\title{
Biodegradable polymer coating promotes the epithelization of tissue-engineered airway prostheses
}

\author{
Toshihiko Sato, MD, ${ }^{\text {a,b }}$ Masato Araki, $\mathrm{MD},{ }^{\mathrm{c}}$ Naoki Nakajima, $\mathrm{PhD},{ }^{\mathrm{d}}$ Koichi Omori, $\mathrm{MD},{ }^{\mathrm{e}}$ and \\ Tatsuo Nakamura, MD ${ }^{\mathrm{a}}$
}

\begin{abstract}
Objective: We have developed a prosthesis that includes a collagen layer for tracheobronchial reconstruction and applied it in a canine model. In previous studies luminal epithelization remained partial or rather slow because of the early disintegration of the collagen layer. We have improved this type of prosthesis by coating the luminal surface with a biodegradable polymer, which serves to protect the collagen layer. The effect of the polymer coating on the epithelization of the luminal surface of the prosthesis was examined.
\end{abstract}

\begin{abstract}
Methods: The main frame consisted of a polypropylene mesh tube, measuring $15 \mathrm{~mm}$ in inner diameter and 30 $\mathrm{mm}$ in length, with reinforcing rings. Collagen extracted from porcine skin was conjugated to this frame. The luminal surface was coated with a polymer, poly (L-lactic-acid-co- $\epsilon$-caprolactone). In 5 beagle dogs the left main bronchus was replaced with this prosthesis, periodic bronchoscopic observations were conducted, and microscopic evaluations were performed.

Results: All dogs survived until they were killed, except for 1 animal in which pneumonia developed, and this animal died at 13 months after replacement. None of the dogs showed adverse complications caused by the prosthesis. Bronchoscopic observations revealed that the polymer remained on the luminal surface for 2 weeks. The luminal surface in 4 dogs was completely covered with ciliated columnar epithelium or nonciliated squamous epithelium, and 90\% epithelization was achieved in $1 \mathrm{dog}$.
\end{abstract}

Conclusions: The biodegradable polymer coating protected the collagen layer and promoted better epithelization. This improved epithelization on the luminal surface could therefore potentially increase the success rates in airway replacement with artificial prostheses. (J Thorac Cardiovasc Surg 2010;139:26-31)

Langer and Vacanti ${ }^{1}$ proclaimed the glorious future of tissue engineering in their review in Science in 1993. Since then, numerous attempts have been made to regenerate the whole trachea, including the cartilage. Kojima and colleagues ${ }^{2}$ successfully created a tissue-engineered trachea with cartilage regenerated from bone marrow cells. Another attempt has been made to regenerate the tracheal cartilage by supplying growth factors. Igai and associates ${ }^{3}$ applied gelatin sponges, which were designed to release basic fibroblast growth factor, into a tracheal defect and observed cartilage regeneration. However, the implantation of such tissue-engineered prostheses has been associated with a poor functional outcome $^{4}$ or only partial tissue regeneration, ${ }^{5}$ and thus its application seems problematic. A polypropylene-framed airway prosthesis conjugated with a collagen layer was developed

\footnotetext{
From the Department of Bioartificial Organs ${ }^{\mathrm{a}}$ and Medical Simulation Engineering, Institute for Frontier Medical Sciences, Kyoto University, Kyoto, Japan; the Department of Thoracic Surgery, ${ }^{\mathrm{b}}$ Kyoto University Hospital, Kyoto, Japan; the Division of Surgical Oncology, Department of Translational Medical Sciences, ${ }^{\mathrm{c}}$ Nagasaki University, Nagasaki, Japan; and the Department of Otolaryngology, ${ }^{\mathrm{e}}$ Fukushima Medical University, Fukushima, Japan.

Received for publication July 24, 2008; revisions received Jan 14, 2009; accepted for publication April 1, 2009; available ahead of print June 18, 2009.

Address for reprints: Toshihiko Sato, MD, Department of Thoracic Surgery, Kyoto University, 54 Kawaharacho, Shogoin, Sakyo-ku, Kyoto 606-8507, Japan (E-mail: tsato@kuhp.kyoto-u.ac.jp).

0022-5223/\$36.00

Copyright (c) 2010 by The American Association for Thoracic Surgery doi:10.1016/j.jtcvs.2009.04.006
}

based on the concept of in situ tissue engineering because regenerating a whole trachea with cartilage remains questionable in terms of durability. The goal of in situ tissue engineering is to create a local environment with a prepared scaffold suitable for tissue or organ restoration. ${ }^{6}$ Collagen is a major component of the extracellular matrix and is known to play active roles in the migration and proliferation of cells. We have designed a prosthesis conjugated with a lyophilized collagen layer to act as a 3-dimensional scaffold and applied it for regeneration of various tissues and organs, including the gastrointestinal tract and peripheral nerves. ${ }^{7-9}$

Previous studies with this type of prosthesis have reported the replacement of the intrathoracic trachea ${ }^{10,11}$ the carinal part of the trachea, ${ }^{12}$ the left main bronchus in a canine model,${ }^{13}$ and the cervical trachea in human subjects. ${ }^{14}$ Previous attempts to replace the airway with this type of prosthesis revealed that the epithelization was either rather slow or incomplete in some cases. The collagen layer possibly disintegrate before host tissue regeneration, and we hypothesized that the collagen layer should be isolated from the luminal space interface and protected against early disintegration for a certain period to achieve better results.

Therefore an improved prosthesis was designed, in which the luminal surface was coated with a biodegradable polymer, poly (L-lactic acid-co- $\epsilon$-caprolactone [ $\mathrm{L}-\mathrm{C}$ copolymer]). L-C copolymer has been applied clinically as 


\section{Abbreviation and Acronym \\ $\mathrm{L}-\mathrm{C}$ copolymer $=$ poly $(\mathrm{L}-$ lactic acid-co- $\epsilon$-caprolactone)}

artificial dura mater material. It is able to seal dura mater defects and reduces the risk of intracranial hypotension. ${ }^{15}$ Sakuma and coworkers ${ }^{16}$ showed that this polymer sheet was applicable as a substitute for the pericardium in an experimental animal model.

The objective of the present study was to clarify whether this type of prosthesis could be applicable for the replacement of the left main bronchus in dogs and produce better results in terms of luminal epithelization.

\section{MATERIALS AND METHODS}

\section{Bronchial Prosthesis}

The collagen-conjugated bronchial prosthesis was made by using a method similar to that described in a previous report. ${ }^{17}$ The prosthesis consists of a framework and a collagen layer. The framework is a fine polypropylene mesh (Marlex mesh; CR Bard, Inc, Billerica, Mass) cylinder, $30 \mathrm{~mm}$ long with an internal diameter of $15 \mathrm{~mm}$, reinforced with 5 rings of polypropylene monofilament string ( $1 \mathrm{~mm}$ in diameter). This cylindrical mesh framework was exposed to a corona discharge at $9 \mathrm{kV}$ for 5 minutes to render its surface hydrophilic. The framework was then placed in a mold, and $2 \%$ collagen solution (Nippon Meat Packers, Inc, Osaka, Japan) was poured into the mold, followed by freeze-drying to form a collagen layer $5 \mathrm{~mm}$ thick around the frame and $2 \mathrm{~mm}$ thick from the luminal surface. The prosthesis was heated at $140^{\circ} \mathrm{C}$ under vacuum pressure for a 24 -hour session of dehydrothermal treatment to induce moderate cross-linkage of the collagen molecules. The L-C copolymer (50:50 mol\%; molecular weight 220,000 d) was prepared by means of conventional ring-open polymerization, according to the method reported by Nakamura and colleagues. ${ }^{18}$ The copolymer solution in 1,4-dioxane was spread onto the luminal surface of the prosthesis twice, followed by overnight drying in a draft chamber. Finally, the prosthesis was sterilized with ethylene oxide gas and stored in a desiccator until use. The schematic and cross-sectional views of the prosthesis are shown in Figure 1.

\section{Animal Experiment}

Five adult beagle dogs weighing 8 to $14 \mathrm{~kg}$ were anesthetized with an intramuscular administration of $15 \mathrm{mg} / \mathrm{kg}$ ketamine hydrochloride and 7 $\mathrm{mg} / \mathrm{kg}$ xylazine and then intubated with an endotracheal tube. Respiration was maintained by using a mechanical ventilator with halothane and nitrous oxide gas. The procedures used for replacement of the left main bronchus were similar to those of a previous study. ${ }^{19}$ Briefly, after the left main bronchus was exposed through the pulmonary-aortic window, a 10-mm segment of the left main bronchus was resected, and the prosthesis was interposed. Both ends of the prosthesis were anastomosed in a telescoped manner with interrupted 3-0 Vicryl sutures (Ethicon, Inc, Somerville, NJ), with the bronchial ends lying inside the prosthesis.

The dogs were appropriately hydrated with extracellular fluid, and 125 mg of hydrocortisone was administered during the operation. Ampicillin was administered intravenously at a dose of $1 \mathrm{~g}$ on the day of the operation and at $0.5 \mathrm{~g}$ daily for 3 days thereafter. Hydrocortisone ( $125 \mathrm{mg}$ ) was administered intravenously for 3 days after the procedure. All of the animals received humane care in compliance with the "Guide for the care and use of laboratory animals" prepared by the Institute of Laboratory Animal Resources, National Research Council, and published by the National Academy Press, revised 1996.

\section{Bronchoscopic Observation}

Bronchoscopic observation was done after achievement of general anesthesia induced with an intramuscular injection of ketamine hydrochloride and xylazine hydrochloride. The condition of the prosthesis after implantation was evaluated with a bronchofiberscope (Model BF1T20; Olympus Optical Co, Ltd, Tokyo, Japan) periodically at 1, 2, and 3 weeks; 1 month; and every 2 months after implantation.

\section{Evaluation of the Prosthesis}

The replaced portion was examined bronchoscopically (Figure 2) and macroscopically after autopsy (Figure 3). Euthanasia was performed with an intravenous injection of $100 \mathrm{mg} / \mathrm{kg}$ ketamine hydrochloride, followed by an autopsy.

Stenosis was defined as a reduction of more than one third of the tracheal lumen cross-sectional area. The state of epithelization was evaluated bronchoscopically and macroscopically to determine whether the mesh underneath the epithelium was exposed. Immediately after macroscopic evaluation, the specimens were placed in $10 \%$ formaldehyde solution, followed by paraffin embedding. Five-micrometer sections were cut and stained with hematoxylin and eosin for light microscopy.

\section{RESULTS}

The outcome of the experiments is shown in Table 1. All the dogs survived the postoperative period without any apparent morbidity. One dog died of pneumonia at 13 months after bronchial replacement. This dog showed no dehiscence of the anastomosis or infection of the prosthesis during the postoperative period until pneumonia developed.

\section{Bronchoscopic and Macroscopic Examination}

Neither stenosis nor excessive granulation was observed in this series of dogs.

The inner lumen was fully covered in $4 \mathrm{dogs}$, and in $1 \mathrm{dog}$ $90 \%$ of the luminal surface was judged to be covered by the epithelium.

Periodic bronchoscopy revealed that the $\mathrm{L}-\mathrm{C}$ copolymer layer remained on the collagen layer for up to 2 weeks (Table 1 ). One week after replacement, the copolymer layer appeared to remain over the entire length, except in $1 \mathrm{dog}$. Two weeks after the replacement, in 4 dogs the copolymer layer had disappeared, whereas in 1 dog the copolymer layer partially remained. Bronchoscopic observations at 1 month after implantation confirmed that the polymer layer and the collagen layer had been replaced by regenerated tissue (Figure 2).

The host tissue incorporated the framework of the prosthesis, and neither erosion nor dehiscence was observed between the framework and the adjacent vessels. No complications, such as hemoptysis or intrathoracic hemorrhage, were observed.

\section{Histologic Findings}

A regenerated ciliated columnar epithelium was observed macroscopically. Near the center of the prosthesis, the proportion of squamous epithelium became larger than that of ciliated columnar epithelium (Figure 3, B 


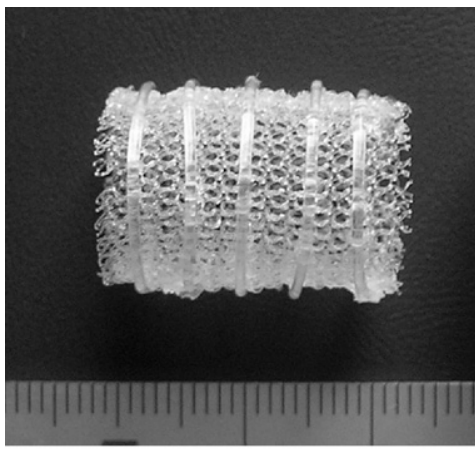

A

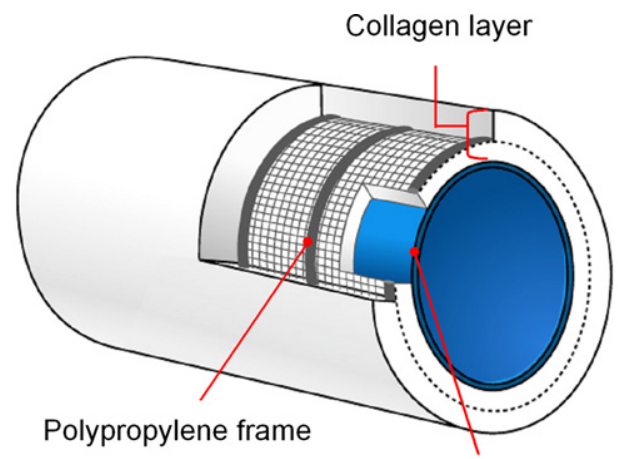

B

L-C co-polymer coating

FIGURE 1. A, The bulk structure of the prosthesis framework: a mesh cylinder reinforced with 5 rings of polypropylene string. B, Cross-sectional schema of the prosthesis conjugated with a collagen layer. The L-lactic acid-co- $\epsilon$-caprolactam ( $\mathrm{L}-\mathrm{C}$ copolymer) layer is colored blue.

and $C$ ). No residual copolymer layer was observed on the resected specimen. No immigration of inflammatory cells was evident under the regenerated epithelium, indicating that the prosthesis was well incorporated without any excessive foreign-body reaction attributable to the copolymer or collagen.

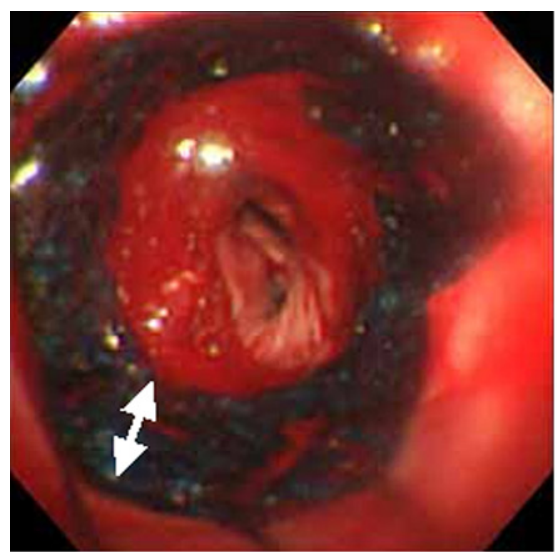

Day 0

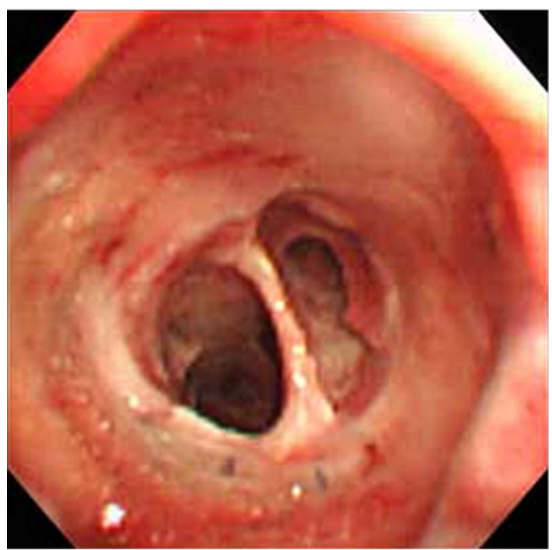

Day 21

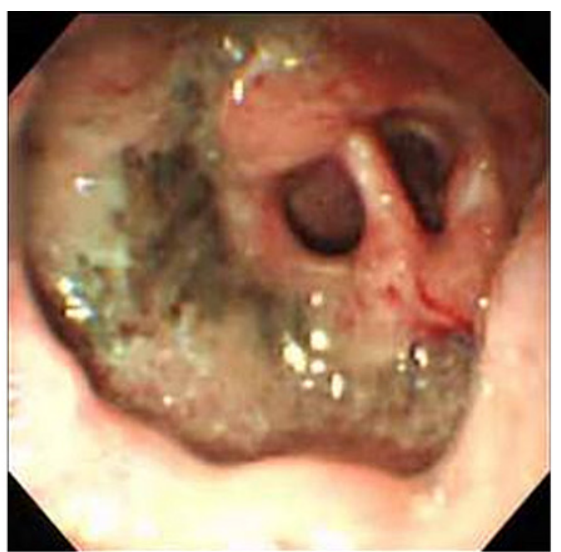

Day 7

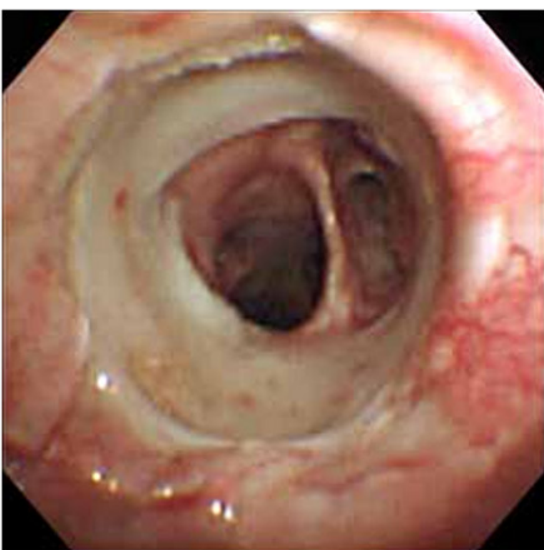

Day 30

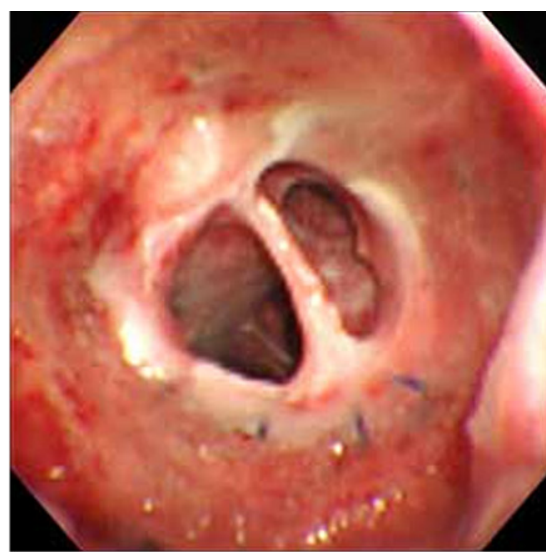

Day 14

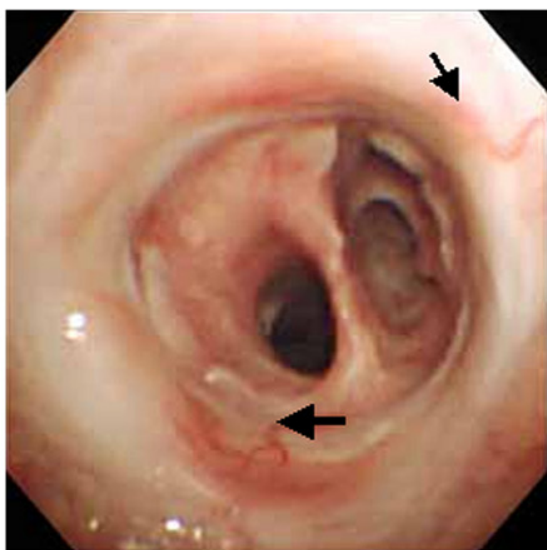

Day 180

FIGURE 2. Periodical bronchoscopic views of the replaced portion after tracheal replacement. At day 0 , the replaced portion can be identified by the bluecolored copolymer layer. At day 7, the copolymer coating remained. At day 14, regenerated reddish tissue could be observed, and the copolymer coating had apparently disappeared. At day 21, the luminal surface was covered with whitish tissue. At day 30 , the luminal surface was completely covered with a smooth epithelium. At day 180, neomucosae with regenerated blood vessels were observed (arrows). 

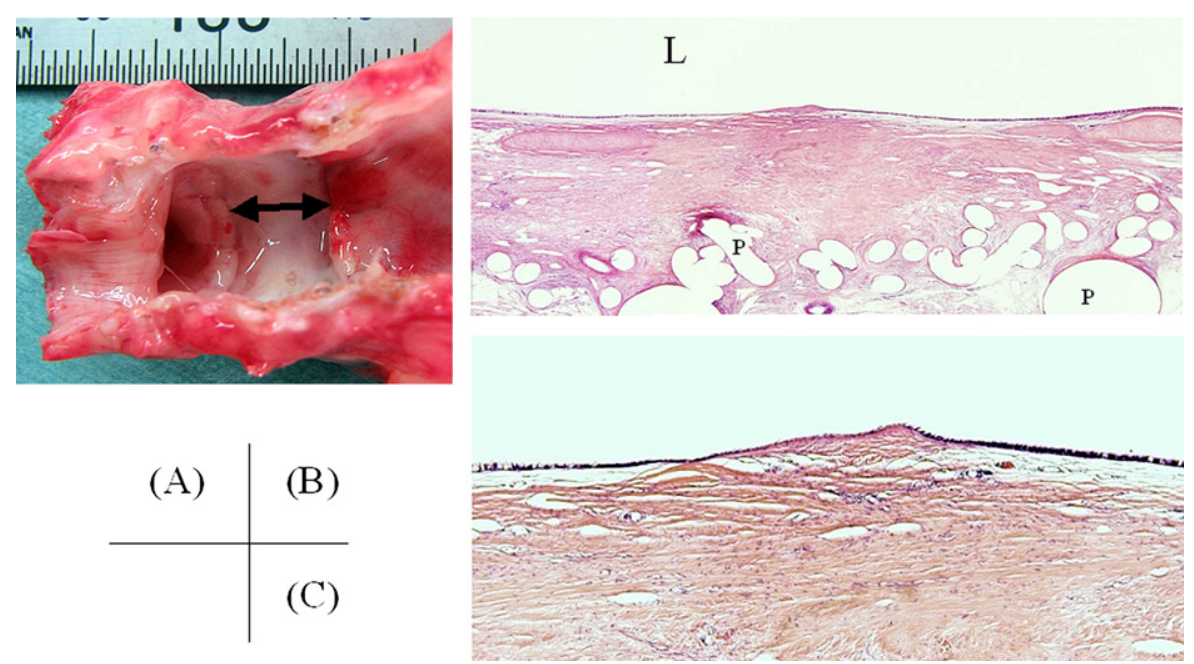

FIGURE 3. A, Macroscopic view of the luminal surface of the prosthesis 18 months after implantation. The arrow shows the regenerated part. The luminal surface is covered with apparently normal mucosa. B, Microscopic examination of a longitudinal section of the regenerated tissue 18 months after prosthesis implantation. The regenerated neomucosa with an epithelial lining is observed on the prosthesis. (Hematoxylin and eosin staining, magnification $10 \times$.) $\mathrm{C}$, Ciliated columnar epithelial cells have grown over the prosthesis, whereas the center of the prosthesis is not as dense as in the peripheral regions. (Hematoxylin and eosin staining, magnification $40 \times$.) $L$, Bronchial lumen; $P$, polypropylene mesh.

\section{DISCUSSION}

A multilayered-type collagen-conjugated prosthesis consisting of collagen and a biodegradable L-C copolymer layer was used to replace the canine left main bronchus. This is the first trial in which $\mathrm{L}-\mathrm{C}$ copolymer was applied in a tissue-engineered airway replacement. In contrast with the previous studies using a collagen-monolayered prosthesis, good results were achieved in terms of epithelization.

Together with major advances in surgical techniques that now enable rather complex forms of bronchoplasty ${ }^{20,21}$ and reconstruction of tracheal defects more than $6 \mathrm{~cm}$ long, ${ }^{22}$ the risk of potentially fatal complications, such as local infection, stenosis, or rupture of the adjacent vessels, ${ }^{23-25}$ still precludes the clinical use of artificial prostheses in this situation. However, because reconstruction of the trachea involving lengthy resections exceeding $4 \mathrm{~cm}$ is associated with a high morbidity rate, ${ }^{26}$ there is a clinical demand for an appropriate prosthesis that can avoid any stretching stress at the anastomosis site. We consider that the conditions required for an ideal prosthesis are (1) adequate durability to maintain a functional airway after implantation, (2) relatively low cost, and
(3) high biocompatibility, thus enabling incorporation into the host tissue. A tissue-engineered collagen-conjugated prosthesis that potentially meets these requirements was investigated. This type of prosthesis is equipped with a polypropylene framework as a substitute for cartilage, which is sufficiently stable to maintain the lumen long after transplantation. Animal experiments have shown that this type of prosthesis has appropriate mechanical properties similar to those of the native trachea. ${ }^{27}$ Observations of a canine tracheal replacement model for more than 5 years has proved that it is durable and free from potentially fatal problems, such as anastomotic leakage or postanastomotic stenosis. ${ }^{28}$ Clinically, Omori and colleagues ${ }^{29}$ have applied this tissue-engineered tracheal prosthesis to the cervical trachea of 8 human patients to date, with a maximum follow-up period of 4 years.

This type of prosthesis is suitable for commercial production in various sizes and lengths at relatively low cost in comparison with biologic transplant grafts and is free from any risk of donor-related infection.

A prosthesis with a foam-type collagen layer results in better tissue incorporation than a prosthesis with an amorphous

TABLE 1. Results of replacement of the left main bronchus

\begin{tabular}{lccccc}
\hline Dog & Stenosis & Epithelization & $\begin{array}{c}\text { State of polymer } \\
\text { coating at day }\end{array}$ & $\begin{array}{c}\text { State of polymer } \\
\text { coating at day 14 }\end{array}$ & Remarks \\
\hline 1 & $(-)$ & $90 \%$ & Full & Partial & Killed at 18 mo \\
2 & $(-)$ & Partial & Disappeared & Pneumonia leading \\
& & & & Do death at 13 months \\
3 & $(-)$ & $100 \%$ & Full & Disappeared & Killed at 18 mo \\
4 & $(-)$ & $100 \%$ & Full & Disappeared & Killed at 17 mo \\
5 & $(-)$ & $100 \%$ & Full & Disappeared & Killed at 17 mo \\
\hline
\end{tabular}


collagen monolayer coat. ${ }^{10,12,17}$ These results indicate that a collagen layer with a 3-dimensional structure potentially provides an appropriate extracellular matrix in which migrating cells can proliferate, thus enhancing tissue regeneration.

Despite these favorable characteristics, the collagen layer is quite fragile and tends to detach either during the surgical procedure or after implantation. A silicone tube has been set inside the prosthesis and then extracted 8 weeks after implantation to protect the collagen layer. This procedure prevented the early degradation of the collagen layer and allowed complete coverage of the prosthesis framework in $90 \%$ of cases. ${ }^{11,12}$

In a previous trial of left main bronchus replacement, ${ }^{13}$ no protectant was applied for the collagen layer because the silicone tube insertion and removal were rather complicated procedures and might cause serious problems. However, complete epithelization was achieved in only 2 of 8 cases.

Based on these observations, a biodegradable polymer was applied to the luminal surface of the prosthesis to protect the collagen layer. The $\mathrm{L}-\mathrm{C}$ copolymer consists of 2 elements: poly-L-lactic acid is rather firm and degrades slowly, and copolymerization with $\epsilon$-caprolactone makes the polymer markedly elastic. The copolymer is designed to disintegrate in about 10 to 20 days, during which time it provides appropriate mechanical support. In the preparation of the prosthesis, the $\mathrm{L}-\mathrm{C}$ copolymer solution was spread on its luminal surface to create an additional layer. This additional polymer layer was able to effectively keep the prosthesis airtight, while also improving its mechanical strength during the implantation procedures. No adverse effects, such as excessive granulation formation or infection, were observed after implantation.

Bronchoscopic observations confirmed that the polymer layer remained at the local site and protected the collagen layer for 7 to 14 days after implantation. The luminal surface was completely covered with epithelium, containing ciliated columnar and squamous epithelium, in 4 of 5 dogs. These results showed a stark contrast to the previous poor outcome in terms of epithelization. ${ }^{13}$ These results suggest that it is important to separate the collagen layer from the luminal interface for 2 weeks to avoid any early disintegration. During this period, the collagen scaffold possibly provides an environment to form subepithelial tissue appropriate for the subsequent epithelization. Clinical experience has demonstrated that a partial replacement of the cervical trachea without protection of the collagen layer required a rather long period (2-22 months) to achieve complete epithelization. No adverse events occurred before the complete epithelization; however, rapid and complete luminal epithelization must be achieved. Although further experiments with replacement using a longer prosthesis are required when considering clinical application, the observations in this study imply that this improved type of prosthesis might allow for rapid and complete epithelization.

\section{CONCLUSIONS}

The present study showed that the biodegradable copolymer poly (L-lactic acid-co- $\epsilon$-caprolactone) can be applied to protect the collagen layer. The biodegradable polymer coating remained until 14 days after implantation. The protection of the collagen layer is important to promote better epithelization on the luminal surface of the artificial bronchial prosthesis in this canine model.

We thank Dr Seijun Fukuda, Dr Toshihiro Okamoto, Dr Fengshi Cheng, and Dr Akihiro Aoyama for their valuable technical assistance. We also thank Mr Jason Lee and Dr Masashi Gotoh for critical reading and helpful suggestions in the preparation of the manuscript.

\section{References}

1. Langer R, Vacanti JP. Tissue engineering. Science. 1993;260:920-6.

2. Kojima K, Ignotz RA, Kushibiki T, Tinsley KW, Tabata Y, Vacanti CA. Tissueengineered trachea from sheep marrow stromal cells with transforming growth factor beta2 released from biodegradable microspheres in a nude rat recipient. J Thorac Cardiovasc Surg. 2004;128:147-53.

3. Igai H, Yamamoto Y, Chang SS, Yamamoto M, Tabata Y, Yokomise H. Tracheal cartilage regeneration by slow release of basic fibroblast growth factor from a gelatin sponge. J Thorac Cardiovasc Surg. 2007;134:170-5.

4. Kojima K, Bonassar LJ, Roy AK, Vacanti CA, Cortiella J. Autologous tissueengineered trachea with sheep nasal chondrocytes. J Thorac Cardiovasc Surg. 2002; 123:1177-84.

5. Macchiarini P, Walles T, Biancosino C, Mertsching H. First human transplantation of a bioengineered airway tissue. J Thorac Cardiovasc Surg. 2004;128:638-41.

6. Ikada Y. In situ tissue engineering: Tissue engineering/fundamentals and applications. Challenges in tissue engineering. Burlington, MA: Academic Press; 2006: 428.

7. Hori Y, Nakamura T, Kimura D, Kaino K, Kurokawa Y, Satomi S, et al. Experimental study on tissue engineering of the small intestine by mesenchymal stem cell seeding. J Surg Res. 2002;102:156-60.

8. Yamamoto Y, Nakamura T, Shimizu Y, Matsumoto K, Takimoto Y, Kiyotani T, et al. Intrathoracic esophageal replacement in the dog with the use of an artificial esophagus composed of a collagen sponge with a double-layered silicone tube. J Thorac Cardiovasc Surg. 1999;118:276-86.

9. Inada Y, Morimoto S, Moroi K, Endo K, Nakamura T. Surgical relief of causalgia with an artificial nerve guide tube: successful surgical treatment of causalgia (complex regional pain syndrome type II) by in situ tissue engineering with a polyglycolic acid-collagen tube. Pain. 2005;117:251-8.

10. Okumura N, Nakamura T, Natsume T, Tomihata K, Ikada Y, Shimizu Y. Experimental study on a new tracheal prosthesis made from collagen-conjugated mesh. J Thorac Cardiovasc Surg. 1994;108:337-45.

11. Teramachi M, Okumura N, Nakamura T, Yamamoto Y, Kiyotani T, Takimoto Y, et al. Intrathoracic tracheal reconstruction with a collagen-conjugated prosthesis: evaluation of the efficacy of omental wrapping. J Thorac Cardiovasc Surg. 1997; 113:701-11

12. Sekine T, Nakamura T, Matsumoto K, Liu Y, Ueda H, Tamura N, et al. Carinal reconstruction with a Y-shaped collagen-conjugated prosthesis. J Thorac Cardiovasc Surg. 2000;119:1162-8.

13. Sato T, Tao H, Araki M, Ueda H, Omori K, Nakamura T. Replacement of the left main bronchus with a tissue-engineered prosthesis in a canine model. Ann Thorac Surg. 2008;86:422-8.

14. Omori K, Nakamura T, Kanemaru S, Asato R, Yamashita M, Tanaka S, et al. Regenerative medicine of the trachea: the first human case. Ann Otol Rhinol Laryngol. 2005; 114:429-33.

15. Yamada K, Miyamoto S, Nagata I, Kikuchi H, Ikada Y, Iwata H, et al. Development of a dural substitute from synthetic bioabsorbable polymers. J Neurosurg. 1997;86:1012-7.

16. Sakuma K, Iguchi A, Ikada Y, Tabayashi K. Closure of the pericardium using synthetic bioabsorbable polymers. Ann Thorac Surg. 2005;80:1835-40.

17. Teramachi M, Nakamura T, Yamamoto Y, Kiyotani T, Takimoto Y, Shimizu Y. Porous-type tracheal prosthesis sealed with collagen sponge. Ann Thorac Surg. 1997;64:965-9

18. Nakamura T, Watanabe S, Shimuzu Y, Hitomi S, Shimamoto T, Hyon S-H, et al. Surgical application of biodegradable films prepared from lactide-caprolactone 
copolymers. Paper presented at: Sixth European Conference on Biomaterials; 1986; Bologne, Italy.

19. Sato T, Tao H, Araki M, Ueda H, Omori K, Nakamura T. Replacement of the left main bronchus with a tissue-engineered prosthesis in a canine model. Ann Thorac Surg. 2008;86:422-8.

20. Kato R, Kakizaki T, Hangai N, Sawafuji M, Yamamoto T, Kobayashi T, et al. Bronchoplastic procedures for tuberculous bronchial stenosis. J Thorac Cardiovasc Surg. 1993;106:1118-21.

21. Bueno R, Wain JC, Wright CD, Moncure AC, Grillo HC, Mathisen DJ. Bronchoplasty in the management of low-grade airway neoplasms and benign bronchial stenoses. Ann Thorac Surg. 1996;62:824-9.

22. Grillo HC, Dignan EF, Miura T. Extensive resection and reconstruction of mediastinal trachea without prosthesis or graft: an anatomical study in man. $J$ Thorac Cardiovasc Surg. 1964;48:741-9.

23. Neville WE, Bolanowski JP, Kotia GG. Clinical experience with the silicone tracheal prosthesis. J Thorac Cardiovasc Surg. 1990;99:604-13.
24. Toomes H, Mickisch G, Vogt-Moykopf I. Experiences with prosthetic reconstruction of the trachea and bifurcation. Thorax. 1985;40:32-7.

25. Deslauriers J, Ginsberg RJ, Nelems JM, Pearson FG. Innominate artery rupture. A major complication of tracheal surgery. Ann Thorac Surg. 1975;20:671-7.

26. Wright CD, Grillo HC, Wain JC, Wong DR, Donahue DM, Gaissert HA, et al. Anastomotic complications after tracheal resection: prognostic factors and management. J Thorac Cardiovasc Surg. 2004;128:731-9.

27. Kawaguchi S, Nakamura T, Shimizu Y, Masuda T, Takigawa T, Liu Y, et al. Mechanical properties of artificial tracheas composed of a mesh cylinder and a spira stent. Biomaterials. 2001;22:3085-90.

28. Nakamura T, Teramachi M, Sekine T, Kawanami R, Fukuda S, Yoshitani M, et al Artificial trachea and long term follow-up in carinal reconstruction in dogs. Int $J$ Artif Organs. 2000;23:718-24.

29. Omori K, Tada Y, Suzuki T, Nomoto Y, Matsuzuka T, Kobayashi K, et al. Clinica application of in situ tissue engineering using a scaffolding technique for reconstruction of the larynx and trachea. Ann Otol Rhinol Laryngol. 2008;117:673-8. 